\title{
Value Creation with Dye's Disclosure Option: Optimal Risk-Shielding with an Upper Tailed Disclosure Strategy
}

\author{
Adam J. Ostaszewski \\ Dept. of Mathematics \\ London School of Economics \\ tel: +44(0) 2079557656 \\ e-mail: a.j.ostaszewski@lse.ac.uk
}

\author{
Miles B. Gietzmann \\ Faculty of Finance \\ Cass Business School \\ tel: +44 (0) 0400286 \\ e-mail: m.b.gietzmann@city.ac.uk
}

(May 2006) This version October 2007

DisclosureRiskShielding-CDAM.tex

CDAM Research Report LSE-CDAM-2007-30

\begin{abstract}
Dye (1985) showed that the optimal disclosure policy, when a manager is randomly endowed with perfect private information, is upper tailed, i.e. the manager only discloses firm value above an appropriate cutoff level. We interpret this strategically as an optimal exercise by management of the embedded formal option to report value. Given any disclosure cutoff level, we value the corresponding option using contingent claims analysis. It is shown that the Dye disclosure cutoff value maximizes the formal option value. We find it to be the minimum possible conditional valuation (conditioned by non-disclosure) which is thus consistent with the intuition that investors should value conservatively. We show how the Dye cutoff can be interpreted as a strike price in a 'protective put' which offers a shield against risk of disclosure of low value. The strategic analysis is further extended by allowing the probability level that the manager is informed to be a choice variable. We show that the manager will never choose to be perfectly endowed with information, and is likely to be more endowed than unendowed. We also present a simple worked example which shows how the total value of the firm changes once the Dye option is formally incorporated.
\end{abstract}

We gratefully acknowledge the comments of Sudipto Bhattacharya, Nick Bingham and Bjorn Jorgensen. 


\section{Introduction}

An early finding in the disclosure literature, provided by Grossman and Hart (1980) and Grossman (1981), has become known as the unravelling result. In a class of models, if rational agents (managers of firms) that hold private information choose to withhold disclosure to interested outsiders such as investors, then those interested parties will follow what we term a 'minimum principle of valuation': they discount the value of the firm down to the lowest possible value consistent with whatever discretionary disclosure is made. Once agents recognize this, they will have an incentive to make full disclosure. The contribution of Dye (1985) was to provide a rationale for why this full disclosure unravelling result might not occur. He showed that the qualitative features of an optimal disclosure policy for management may take the form of a policy dependent on a cutoff in which management disclose only if the information is sufficiently good, otherwise they do not disclose. The new friction he introduces to explain movement away from the unravelling scenario is that investors might not be able to distinguish agents holding undisclosed information from agents not holding the undisclosed information. In such a setting investors seeing non-disclosure have to temper their inferences concerning the likelihood of a manager having observed bad news and choosing not to disclose (that is choosing to "sit on" bad information) by the fact that non-disclosure may have arisen because instead management were in fact uninformed. This provides us with one clear rationale for why management may choose not to disclose information. Jung and Kwon (1988) developed the Dye result by presenting comparative statics, for instance showing how the level of the cutoff depends on $q$ (the probability that the manager is informed). Intuitively speaking, they explain how, if a manager is informed with a higher probability $q$ (closer to one), it is rational for the investor seeing non-disclosure to assign a higher (conditional) probability that the manager was informed of a poor (below cutoff) realization, rather than that the disclosure arose from lack of information.

We interpret management's choice of whether to disclose discovered value or not strategically, as an optimal exercise of an embedded option to report value. Using contingent claims analysis, we show that the Dye cutoff maximizes option value. We find it to be the minimum possible conditional valuation (conditioned by non-disclosure), which is thus consistent with the intuition that investors should value conservatively. Thus our 'minimum principle of valuation' holds in this new setting. We also show how the Dye cutoff can be interpreted as a strike price in a 'protective put' which offers a shield to stock-holders against risk of disclosure of low value.

Further important insights result if the analysis is extended to allow $q$ (or, equivalently, in Dye's notation: $p$, where $q=1-p$ ) to be identified explicitly as a choice variable that influences what we call 'total' firm value (defined in Section 2). Within this optimizing framework we are able to provide a link between disclosure incentivization and firm value maximization, which some authors have commented is missing in the literature (see for instance Christensen and Feltham (2003)).

We provide the linkage between disclosure and firm value after noting that, by simple inspection of a payoff formula at the disclosure date (which we term the interim reporting date), one can see immediately a structure isomorphic to a financial option contract. Indeed, as shall be explored in more detail below, it aids intuition to recognize that the Dye disclosure option is equivalent to the purchase of a protective put. When management choose $p$ to maximize firm value, it is shown below that this involves choosing the optimal strike price for the Dye option. That is, managers select an optimal disclosure cutoff value in two steps: maximizing over cutoffs given $p$, and then maximizing over $p$. An interesting feature of this formal analysis is that it is never optimal for management to be completely informed $(p=0, q=1)$, so optimal choice avoids 
low firm value realizations always being exposed at an early date.

In order to relate our research to some other recent papers we now comment on their differing research agendas. One stream of research starting with Penno (1997) has developed new insights under the assumption that rather than the manager perfectly observing firm value with probability $q$, instead the manager observes firm value imperfectly - with superimposed noise. Thus in this class of model the precision (noise level) of the signal that the manager observes becomes a critical determinant of disclosure strategy. Here noise level is measured by the inverse variance of a normally distributed noise $\varepsilon$ (i.e. precision $\rho=1 / \sigma_{\varepsilon}^{2}$ ). In the current work we are interested in an optimal choice $\hat{q}$ for $q$, given a distribution $F$ of future firm value, leading to the dependence $q=\hat{q}(F)$. Thus one way our model could be further extended would be to allow noisy observation and derive the corresponding optimal $q$ as a function also of the superimposed 'noise level'. Accordingly we should have $q=\hat{q}(F, \rho)$, opening perhaps the possibility of making an optimizing choice over $\rho$. Note that this differs from Penno's existing approach, since he seeks to relate the intensity of disclosure (for definition see Section 4) to the probability of noisy observation $q$ when $q=q(\rho)$, i.e. $q$ is regarded as function of the 'noise level'; however, the functional forms $q(\rho)$ considered by Penno are exogenously imposed and so it remains unclear what connection these results may have with an optimal selection problem for an endogenously available choice of $q$ given $\rho$. Further extensions of this noisy observation literature ${ }^{1}$ involve allowing multidimensional $^{2}$ signalling, as in Hughes and Pae (2004), or allowing repeated independent observation (sampling), as in Pae (2005).

Another stream of research starts explicitly by studying disclosure in a dynamic setting. For instance, Cosimano, Jorgensen and Ramanan (2002) and also Einhorn and Ziv (2005a) show how multi-period considerations affect disclosure strategy. In a related fashion Einhorn and Ziv (2005b) also incorporate managerial inefficiencies with inter-temporal observation and disclosure; however, this class of models does not endogenize the choice of information structure $(q)$ directly in a firm-value relevant setting, as we do here.

Our approach is to commence by revisiting the original Dye paradigm of uncertain acquisition of perfect (i.e. non-noisy) observation of firm value in order to create the simplest possible setting to study other strategic features such as: behaviour incentivization at the disclosure moment, interactions between disclosure and insider trading (which hints at a possible approach to such matters as strategic manipulation of 'esop' dates and of stock repurchasing), and principle-agent type interactions between disclosure policy and managerial effort provision.

The structure of the paper is as follows. Section 2 contains our main results. In it we begin by describing the Dye model, focusing at first on the naturally occurring underlying call-like structure; we then define 'total value', after a discussion of the trading opportunities arising from the manager's inside information, and state our main theorems characterizing the Dye cutoff, including as corollary the identification of the 'protective put'; that is followed by a qualitative discussion of additional value creation and of connections with risk-management. In Sections 3 and 4 we derive additional properties of the optimal cutoff, generalizing and extending the results given in Jung \& Kwon (1988). For instance; Section 4 considers the 'intensity of disclosure' as a function of the information type, defined to be the ex-ante risk-neutral probability that a disclosure occurs, and it is shown that the intensity is below $\hat{q}=\hat{q}(F)$ if and only if $\hat{q}$ itself is below the Conjugate Golden Mean, $\phi$, circa 0.62 . In Section 5 we identify a particularly simple lower bound for the optimal Dye cutoff $\gamma(\hat{p})$, when the density function is a power function on the

\footnotetext{
${ }^{1}$ See also the work of Tzur and Yaari (1999), Baginski et al. (2000) and Campbell et al (2003).
} 
range $[0, \mu]$, the bound being in terms of the power index. Concluding comments are presented in Section 6. A proof of the Marginal Value Theorem of Section 2 is given in the Appendix.

\section{Modelling disclosure and total value, results and implications}

We assume the Dye (1985) stylized setting in which there are three successive dates: the 'ex-ante' date, the 'interim' reporting date, and the 'terminal' date. At the ex-ante date the manager of a firm and a risk-neutral investor have common prior beliefs regarding the distribution of the terminal date valuation, $X$, of the firm on an outcome interval $\Omega_{\text {value }}$, which we normalize to be the unit interval $[0,1]$. Here one can interpret 0 as the minimum possible value for the firm and 1 to be the maximum possible value. At this ex-ante date the expected terminal value (the first moment of the distribution) is $\mu$. However, shortly afterwards it is common knowledge that, just prior to the interim reporting date, the manager has the chance to privately observe (discover) the realization of the true firm value, denoted $x$. Thus the manager's informational endowment ('type') is modelled with an event space $\Omega_{\text {info }}=\{u, i\}$, and the manager remains uninformed (of type $u$ ) with probability $p$, and will be perfectly informed of the firm value with probability $(1-p)$. It is assumed that the endowment status (type) of the manager that may occur and the realization of firm value are independent random variables. If the manager receives the signal $x$, the manager chooses either to make a credible announcement of the value of the observed signal, or to make no disclosure. In the case that the manager does not receive a signal, it is assumed that the manager cannot make a credible claim of being uninformed at this stage. Hence, when the investor sees no disclosure from the manager, it is common knowledge that this could arise either because the manager did not receive any new information concerning firm value (an event with probability $p$ ), or because the manager chose not to disclose information seen.

Thus we may define two event subspaces as:

- the informed subspace $I=\{i\} \times[0,1]$ and

- the uninformed subspace $U=\{u\} \times[0,1]$.

The 'full event space' is thus $\Omega_{\text {info }} \times \Omega_{\text {value }}=\{i, u\} \times[0,1]$ and carries a product measure $\mathbb{P}$ simply constructed from the two independent random variables: $e$ the information endowment (type) with $e \in\{i, u\}=\{$ informed, uninformed $\}$ and the terminal firm value $X$, distributed over $[0,1]$. We assume, as does Dye, that $X$ has a distribution function $F(x)$, with mean $\mu$, generated from a continuous density $f(x)$ satisfying $f(x)>0$ for $x>0$. The product measure over $\omega$ in $\Omega_{\text {info }} \times \Omega_{\text {value }}$ is defined by:

$$
\begin{aligned}
& d \mathbb{P}(\omega)=p d F(x)=p f(x) d x, \text { if } \omega=(u, x), \text { and } \\
& d \mathbb{P}(\omega)=(1-p) d F(x)=(1-p) f(x) d x, \text { if } \omega=(i, x),
\end{aligned}
$$

cf. Christensen and Feltham (2003) page 504. We define the meaning of 'asset' terminal value to be the random variable $\mathbb{X}$ on the 'full event space' obtained by setting $\mathbb{X}((e, x))=x$. This models the value of the firm as it becomes known to all market participants at the 'terminal date'. Note the important distinction between $\mathbb{X}$ defined on $\Omega_{\text {info }} \times \Omega_{\text {value }}$ and the random variable $X$ defined only on $\Omega_{\text {value }}=[0,1]$.

In the Dye disclosure model the manager selects a disclosure cutoff $t \in[0,1]$ so that, for states of nature in $I(t)=\{i\} \times[0, t)$, the discovered value is not disclosed, whereas, for states in $D(t)=\{i\} \times[t, 1]$, values are truthfully disclosed and believed by the recipients. Hence, in the event of non-disclosure at the interim reporting date, the investors recognize that their 
information identifies the state of nature to be in the subspace $N(t)=U \cup I(t)$, and they value the company according to the corresponding conditional risk-neutral expectation, namely:

$$
V=V(t)=E_{\mathbb{P}}[\mathbb{X} \mid N(t)]=\frac{E_{\mathbb{P}}\left[\mathbb{X}\left(\mathbf{1}_{U}+\mathbf{1}_{I(t)}\right)\right]}{E_{\mathbb{P}}\left[\left(\mathbf{1}_{U}+\mathbf{1}_{I(t)}\right)\right]} .
$$

We refer to this as the 'uninformed' investor valuation. Notice that since $E_{\mathbb{P}}\left[\mathbb{X} \mathbf{1}_{I(t)}\right]<\mu E_{\mathbb{P}}\left[\mathbf{1}_{I(t)}\right]$, for $0<t<1$, and $E_{\mathbb{P}}\left[\mathbb{X} \mathbf{1}_{U}\right]=\mu E_{\mathbb{P}}\left[\mathbf{1}_{U}\right]$ we have

$$
V(t)<\mu \text {. }
$$

To see the emerging role of $V(t)$ in the context of ex-ante risk-neutral valuation, consider first the implications of valuing ex-ante the following contingent claim which we term the investor's call $\mathbb{C}_{t}$. This call-like claim refers to information sets of an investor at the interim date and uses an arbitrary valuation $t$ (with $0<t<1$ ) when no disclosure occurs.

Definition 1: For any $t$ with $0<t<1$, define the claim $\mathbb{C}_{t}$ by the payoff:

$$
\mathbb{C}_{t}(\omega)=\left\{\begin{array}{lc}
t, & \text { if } \omega \in N(t), \\
x, & \text { if } \omega=(i, x) \in D(t) .
\end{array}\right.
$$

For a graphical interpretation, we restrict attention to $\{i\} \times \Omega_{\text {value }}$ identifying this set with $[0,1]$, thus obtaining the following claim by restriction to $\Omega_{\text {value }}$ :

$$
C_{t}(x)=\max \{X(x), t\}= \begin{cases}t, & \text { if } x<t \\ x & \text { if } x \geq t\end{cases}
$$

The claim $C_{t}(x)$ is illustrated in Figure 1 and has the hockey-stick shape typical of a call-option (translate of a call).

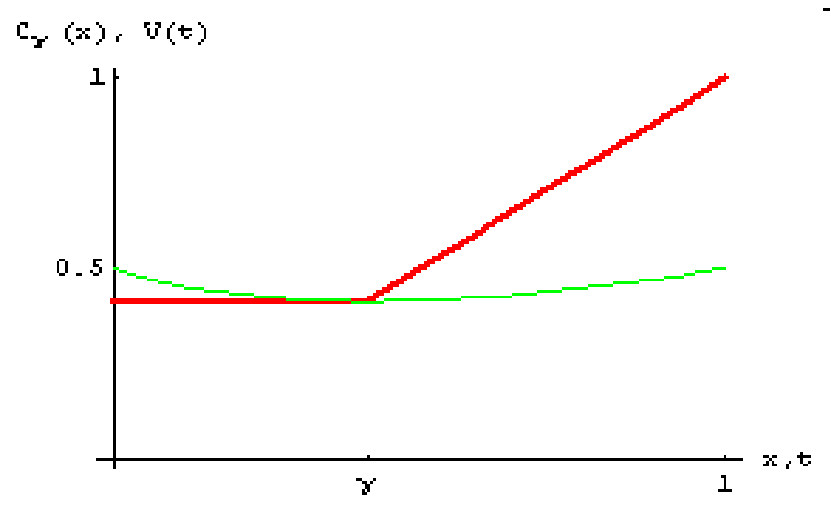

Figure 1. Call-like valuation $C_{\gamma}(x)$ versus realized value $x$ (thick); valuation conditional on non-disclosure $V(t)$ versus cutoff $t$ (faint).

In the language of characteristic functions we have that

$$
\mathbb{C}_{t}=\mathbb{X} \mathbf{1}_{D(t)}+t \mathbf{1}_{N(t)}
$$


It is useful to refer to a related claim, namely:

$$
\mathbb{C}_{t}^{V}=\mathbb{X} \mathbf{1}_{D(t)}+V(t) \mathbf{1}_{N(t)}
$$

for arbitrary $t$. The partition

$$
\mathbf{1}=\mathbf{1}_{U}+\mathbf{1}_{I(t)}+\mathbf{1}_{D(t)},
$$

and the defining property of conditional expected value, displayed in (2.1), permit us to compute the ex-ante expected value of this last claim to be:

$$
E_{\mathbb{P}}\left[\mathbb{C}_{t}^{V}\right]=E_{\mathbb{P}}\left[\mathbb{X} \mathbf{1}_{D(t)}+V(t) \mathbf{1}_{N(t)}\right]=E_{\mathbb{P}}\left[\mathbb{X} \mathbf{1}_{D(t)}+\mathbb{X}\left(\mathbf{1}_{U}+\mathbf{1}_{I(t)}\right)\right]=E_{\mathbb{P}}[\mathbb{X}]=\mu
$$

for any $t$ with $0<t<1$. Hence the following characterization is immediate.

Proposition (No-arbitrage characterization of the Dye cutoff). The unique cutoff $\gamma$ such that $\gamma=V(\gamma)$ is that cutoff $t$ for which

$$
E_{\mathbb{P}}\left[\mathbb{C}_{t}\right]=\mu
$$

Thus the investor's call $\mathbb{C}_{\gamma}$, where $\gamma=V(\gamma)$, which corresponds to the Dye cutoff, creates no additional ex-ante expected value to the investor. On reflection, this should be no surprise, in view of the operation of the law of iterated expectation. Pursuing our quest to find ex-ante value arising from the presence of the manager, we turn to the information sets of the manager at the interim date. When the manager adopts a cutoff $t$ and no disclosure is made the manager knows that either the event $U$ has occurred or that the discovered value is below the cutoff $t$. In the first case the manager's knowledge of the value of the firm at the interim date is the same as ex-ante, so we will regard it as being represented on $U$ by $\mathbb{X} \cdot \mathbf{1}_{U}$. The expected value of this random variable is $\mu$ which is more than $V(t)$. In the second case the manager knows the interim market valuation to be $V(t)$, possibly an improvement over the privately known, undisclosed value (certainly an improvement if $t=\gamma$ ). In either case there is here apparently a source of additional value. It is convenient to formalize these observations in the form of the following contingent claim. The term 'total value' describing it will soon become clear.

Definition 2: For any $t$ with $0<t<1$, the firm's total value, given $t$, refers to the conditional valuation $V(t)$ at the 'interim reporting-date' defined in (2.1) and is defined to be the random variable $\mathbb{W}=\mathbb{W}(t)=\mathbb{W}(t, \omega)$, where

$$
\mathbb{W}(t)=\mathbb{X} \cdot \mathbf{1}_{U}+V(t) \cdot \mathbf{1}_{I(t)}+\mathbb{X} \cdot \mathbf{1}_{D(t)}
$$

We assert that on the uninformed subspace $U$ the manager, knowing (with probability $p$ ) that he is uninformed, should subjectively value the firm at $\mu$. This is justified by considering a notional trade in which the manager buys one share at the interim reporting date which he buys $^{2}$ for $V=V(t)$ (the new market price when the market sees no disclosure), and then sells it back to the market at the terminal date. The conditional expected value of the trade at the interim reporting date (conditional on him not knowing the true value) is $\mu-V$. The ex-ante expected value of this trade is thus

$$
p(\mu-V(t)) .
$$

\footnotetext{
${ }^{2}$ This is a simplification: with $N$ shares outstanding the price is $V / N$ per share, the expected value per share purchased is $\mu / N$ and the expected gain per share purchased is $(\mu-V) / N$.
} 
The expression just displayed is thus the value of a notional or 'formal' option and corresponds to trading what we call the 'manager's contingent claim' at the interim reporting time. This opportunity to trade arises only at the interim reporting date and not any earlier. As just indicated, the value of the expression can in principle be replicated by an insider trading strategy; the value might also be replicated by other means, such as a corresponding equivalent reward to the manager (expressly stated, or selected by way of an 'esop'), or via a stock-repurchase. Theorem 3 below relates the minimization of $V(t)$ over $t$ to the maximization of an objective function for the manager. The theorem thus entitles us to analyse disclosure by a firm as though this formal option was realizable by some mechanism (see the discussion in the following subsection).

\subsection{Consequences of insider trading: components of total value}

From the discussion above it is clear that any model of disclosure value will be intimately related to a model in which managers have insider dealing possibilities. Indeed without this being the case, it is inconceivable that the strategically active manager would have anything of value to communicate. This, however, opens a critical modelling issue, since it would be unrealistic to assume that a manager has unlimited insider dealing possibilities. A key modelling issue then is how to allow for insider information based activity, which could lead to non-disclosure having value, and at the same time place limited restrictions on such behaviour, so as to capture imperfect institutional constraints that may be in place. Thus it is important here to stress exactly what sort of 'insider' dealing possibilities managers have in the current model. For us the only source of insider dealing possibilities is that the manager "knows that he/she does not know". That is, dealing opportunity occurs when non-disclosure results from not receiving a signal on firm value. Seeing investors lower their valuation of the firm because of non-disclosure, the manager who has not been informed, knows that this revaluation is unwarranted because non disclosure did not result from a signal of below cutoff value, but instead from no signal having been received. As is assumed in the original Dye model, the manager cannot credibly signal non-receipt of information at this stage. To reiterate this point: when we talk about insider dealing in this paper, we are only referring to the above situation, not the more controversial case of the manager receiving a lowvalue signal and then trading on that news without having made a disclosure. Thus in our model the insider dealing strategy could be implemented, for instance by the company repurchasing stock following non-disclosure. In such a setting it is unlikely that the securities regulators would find against the company, since the firm has not traded on a new signal of firm value. For a defence of assuming an even stronger form of insider dealing in disclosure models see Baiman and Verrecchia (1996).

Returning to how participants (investors) form inferences in such a setting, we note that in principle a market participant would be able to infer immediately the manager's information type (endowment) from the manager's trading strategy, if it was observable and known to follow a deterministic (pure) strategy based on his information. But this inference is invalid if either the trading is not observable, or it is known that the manager pursues an optimized, mixed strategy of buying and selling on the market, purposefully attempting to conceal some, or all, of his information (perhaps successfully most of the time). Indeed such activity might be modelled by multiple buying and selling in the time interval after the interim date. In this paper we are concerned with the consequences of being able to realize the value of the 'manager's contingent claim'. We therefore do not attempt to model any particular market mechanism, and are content

to note a very simple facilitating mechanism which would require the manager to enter into a onestage 'obligatory auction' against an uninformed trader in which bids are made simultaneously. 
This ensures that the payoff in equilibrium to the manager's bid is ex-ante expected to be the simple expression:

$$
p(\mu-\gamma)
$$

In summary, to permit the expected value (2.6) to be replicated in trading strategies, the manager may need to be empowered to trade on private information with, for the example, the obligation to share a proportion of the gains with the investor. Additionally, in the interests of fairness (but not for strategic reasons), the manager here is not allowed to short the stock. This aligns the interests of the manager with those of the investor, but a similar incentive effect could be achieved by offering the manager payment of some proportion of $\mu-V$ at the interim date, where $V=V(t)$ is the price of the stock at the interim date. In the former case it should be noted that the shareholders of the company are accordingly deemed to hold two financial instruments: the underlying claim to $X$ and the claim (right and obligation) to a share in the manager's contingent claim (be it gain, or loss). These two components taken together define what we mean by the expression 'total value' in Definition 2 above. An obvious extension to this model would be to introduce agency theory considerations; however, in the agency context the 'first best solution' is usually trivial, whereas here it is not so, hence this paper concentrates on characterizing the features of the first best. ${ }^{3}$

For a discussion of the pros and cons of insider trading see Leland (1992); note especially that Leland's model implies an increase on average in the value of shares when insider trading is permitted which finding is in keeping with our positive additional value creation.

An important implication is that the expected total value of the firm (when investors are given a positive proportion $\alpha$ of the manager's contingent claim) is now lifted from $\mu$ to

$$
\mu+\alpha p(\mu-V(t))
$$

For simplicity we have assumed that $\alpha=1$.

Thus the manager who anticipates the uniformed investor valuation (2.1), in response models the total value which he can control, or can 'create', by the random variable $\mathbb{W}$, as compared to $\mathbb{C}_{t}^{V}$ defined in (2.3). When the manager's interests are aligned with investors, the manager acts so as to maximize the ex-ante valuation

$$
W(t):=E_{\mathbb{P}}[\mathbb{W}(t)]
$$

This is the manager's objective function alluded to earlier.

\footnotetext{
${ }^{3}$ To set the matter of simplicity in perspective, note that, if the manager is informed at the interim date and the circumstance is that he makes no disclosure, then the market value of the share is $V$, but the manager knows the realization is $X=x<V$. That is, an over-valuation of $V-x$ occurs. The manager can notionally realize this value increment and execute an arbitrage opportunity by being permitted to enter a short sale. In contrast, to the previous option to gamble, this particular trade may be deemed as taking unfair advantage of the market. When the distribution of firm value is uniform, the ex-ante expected value of the arbitrage opportunity per unit share is

$$
(1-p) \gamma\left(\gamma-\frac{\gamma}{2}\right)
$$

where $\gamma=\gamma(p)$. The formula expresses the fact that the over-valuation occurs with probability $q \gamma$ and the expected gain on a single short position is the difference between the interim price $\gamma$ and the expected low realization which is the mid-point value $\gamma / 2$. As a function of $p$ this expression is again zero at the end-points $p=0$ and $p=1$ and is concave. Permitting this kind of trade would not alter the manager's insider gains qualitatively. The interests of fairness and simplicity warrant exclusion of this extra term from consideration.
} 
We now employ contingent claim analysis in place of the notional trading strategy presented earlier to compute $W(t)$. Using the partition (2.4) for the purpose of eliminating $\mathbf{1}_{D(t)}$, we obtain a decomposition of $\mathbb{W}$, which has an immediate interpretation, namely as a 'shortfall-or-nothing' option (defined on the informed subspace) plus 'asset', as follows:

$$
\mathbb{W}(t)=(V-\mathbb{X}) \cdot \mathbf{1}_{I(t)}+\mathbb{X}
$$

The terminology suggests that $V$, the uninformed investor valuation, is a 'target' value, and $V-\mathbb{X}$ measures the shortfall from the target. We stress at this juncture, because it will be critical later when we identify the underlying 'protective put', that the manager, by not disclosing value, protects investors from receiving a low market value when the above shortfall could be large. (Recall that, in the event of non-disclosure, shares will be traded at $V(t)$ at the interim date.)

Now the conditional valuation definition of $V=V(t)$ (where $t$ is any cutoff which the manger is known to be using) is such that

$$
E_{\mathbb{P}}\left[\mathbb{X} \cdot \mathbf{1}_{U}+\mathbb{X} \cdot \mathbf{1}_{I(t)}\right]=E_{\mathbb{P}}\left[V\left(\mathbf{1}_{U}+\mathbf{1}_{I(t)}\right)\right]
$$

and re-arranging we obtain:

$$
E_{\mathbb{P}}\left[(V-\mathbb{X}) \cdot \mathbf{1}_{I(t)}\right]=E_{\mathbb{P}}\left[(\mathbb{X}-V) \mathbf{1}_{U}\right]
$$

But the 'uninformed excess' claim is given by

$$
E_{\mathbb{P}}\left[(\mathbb{X}-V) \mathbf{1}_{U}\right]=p(\mu-V)
$$

Hence we have valued the (manager's) 'informed shortfall-or-nothing' option struck at $t$ :

$$
E_{\mathbb{P}}\left[(V-\mathbb{X}) \cdot \mathbf{1}_{I(t)}\right]=p(\mu-V) .
$$

Consequently, in agreement with valuations based on trading, we have

$$
\begin{aligned}
W(t) & :=E_{\mathbb{P}}[\mathbb{W}(t)]=E_{\mathbb{P}}\left[(V-\mathbb{X}) \cdot \mathbf{1}_{I(t)}+\mathbb{X}\right] \\
& =E_{\mathbb{P}}\left[(V-\mathbb{X}) \cdot \mathbf{1}_{I(t)}\right]+E_{\mathbb{P}}[\mathbb{X}] \\
& =p(\mu-V)+\mu
\end{aligned}
$$

Since $\mu$ is the ex-ante value of the company when no opportunity exists of early value-discovery (that is, prior to the interim reporting date), the equation (2.10) identifies the 'disclosureendowment' option (with cutoff $t$ ) as having the risk-neutral valuation:

$$
U=U(t)=p(\mu-V(t))
$$

which is positive for $p$ positive, for $0<t<1$, by (2.2). We have thus proved:

Theorem 1 (Valuation of the disclosure-endowment). If the manager's objective is to maximize over $t$ the expected 'total value' $E_{\mathbb{P}}[\mathbb{W}(t)]$, where the total value $\mathbb{W}(t)$ is defined by (2.5), then this amounts to maximizing the expression

$$
\mu+p(\mu-V(t))
$$

where $p$ is the given positive probability of non-discovery and $\mu$ is the unconditional expectation of the firm's terminal value. 
Since shares may be traded at the ex-ante date, we have assumed that the manager has been incentivized to maximize the ex-ante stock value. This value is given by the risk-neutral valuation $W(t)=E_{\mathbb{P}}[\mathbb{W}(t)]$. His objective is thus to maximize $U(t)$ over $t$, which in turn requires him to minimize $V(t)$ over $t$. Theorem 1 thus goes some way towards offering an ex-ante justification for the assumption that the manager seeks to maximize firm value at the interim reporting date. Our next result leads to an identification of the unique optimal choice of $t$.

Theorem 2 (Characterization of Marginal Values). Relative to variation in the cutoff $t$, the marginal values of $U, V, W$ are given by

$$
d U(t)=d W(t)=-p d V(t)=p q(V(t)-t) \frac{d F(t)}{\mathbb{P}[N(t)]},
$$

where $\mathbb{P}[N(t)]$ denotes the probability of the event $N(t)$ under the measure $\mathbb{P}$.

Comment. The formula (2.12) exhibits the trade-off between the incremental value of disclosure, as represented by the $t$ term, and the value of non-disclosure as represented by the $V(t)$ term. This interpretation brings our approach into coincidence with Dye's.

The proof is given in the Appendix. An immediate consequence is the following key result.

Theorem 3 (Minimum Principle for the Dye cutoff). The function $V(t)$ achieves its minimum on $[0,1]$ at the same location as the function $W(t)$ achieves its maximum on $[0,1]$, namely at the unique solution $t=\gamma$ of

$$
V(t)=t,
$$

that is at the Dye cutoff.

Necessarily $\gamma<\mu$, and hence the optimized formal 'information-endowment' option is in fact the 'Dye disclosure option' and has the positive value

$$
p(\mu-\gamma)
$$

Comment. We stress that the theorem does not depend on insider trading assumptions. It merely identifies where certain functions, namely $V(t)$ and $W(t)$ have extrema. When no disclosure occurs, in the absence of any further information, investors would wish on intuitive grounds to value the stock conservatively, that is as low as possible. The theorem asserts that this minimum valuation occurs when $t=\gamma$, that is, as though the manager acted so as to maximize $W(t)$. Rather than have $W(t)$ represent some form of shadow price, we prefer to interpret the formal option as a realizable gain (or loss) to a manager empowered to trade.

We show below in Subsection 2.3 (c) that the minimum characterization of $\gamma$ (i.e. as the minimizer of $V(t)$ over $t$ ) can be re-interpreted in the language of risk-management afforded by the gain-to-loss ratio known as the Omega function introduced in Keating and Shadwick (2002a) and Keating and Shadwick (2002b).

The optimal disclosure cutoff (above which a value, if discovered, is reported) which maximizes expected firm value prior to the interim reporting date was derived by Dye (1985) as an equilibrium strategy by implicit reference to the indifference principle (i.e. to the manager's indifference between non-disclosure and disclosure at the cutoff). We note this observation is made explicit in Cosimano, Jorgensen and Ramanan (2002). As we have just pointed out, Theorem 3 characterizes Dye's cutoff value $\gamma(p)$ as the cutoff which is set equal to the minimum valuation conditional on 
non-disclosure (minimum taken over all disclosure cutoffs). Thus the uninformed valuation, i.e. the minimum conditional valuation, becomes the investor's equilibrium proxy for unreported value. This minimum principle of valuation operating in the Dye model is thus in keeping with Grossman (1981), wherein the uninformed parties discount the value of the firm to the lowest possible value consistent with whatever discretionary disclosure is made. One interesting implication of our minimum principle is equilibrium stability: suppose the investor first guesses a cutoff equal to the mean $\mu$ of $X$ and responds with a conditional valuation $V(\mu)$, then the manager will adjust his/her cutoff to $V(\mu)$; the investor in turn should respond with a conditional valuation of $V(V(\mu))$ and so on, and this sequence of adjusted responses arising from the initial guess will provably converge to $\gamma(p)$ (indeed is liable to converge reasonably quickly, under mild distributional assumptions).

Proof of Theorem 3. The characterization of the optimal cutoff follows from Theorem 2. The uniqueness of the solution to $V(t)=t$ is well-known (Dye, Jung \& Kwon). Since the solution $\gamma$ to $V(t)=t$ is necessarily in the interval $(0,1)$, we have $\gamma=V(\gamma)<\mu$ by $(2.2)$.

Corollary (Implicit Protective Put) If $\gamma$ is the solution to $V(t)=t$, then

$$
E_{\mathbb{P}}\left[(V-\mathbb{X})^{+} \cdot \mathbf{1}_{I}\right]=p(\mu-\gamma) .
$$

Comment. Thus the Dye-disclosure option value $p(\mu-\gamma)$ is seen to be notionally a put option value in the informed state; that is, $(V-\mathbb{X})^{+} \cdot \mathbf{1}_{I}$ is the implicit protective put contract (given to the manager) on the 'asset' value $\mathbb{X}$ falling below the strike $V(\gamma)=\gamma$ in the notional context of an informed manager offering full disclosure. We stress that we have left open the mechanism selection by which the manager participates in this value creation .

Proof. If $t$ is selected to be the Dye cutoff $\gamma$, then in fact we have, by appeal to (2.9)

$$
E_{\mathbb{P}}\left[(V-\mathbb{X})^{+} \cdot \mathbf{1}_{I}\right]=E_{\mathbb{P}}\left[(V-\mathbb{X}) \cdot \mathbf{1}_{I(\gamma)}\right]=p(\mu-\gamma),
$$

because, by definition, $V(\gamma)=\gamma$ and $I(\gamma)=\{i\} \times[0, \gamma)$.

The Informed-put is thus interpreted as 'protective' insurance against the asset value $\mathbb{X}$ falling below the uninformed investor valuation. In conclusion, the ex-ante expected 'total value' of the firm in the presence of an endowment of imperfect information (for an exogenously given positive $p$ ) is given, as in (2.10), by the risk-neutral valuation:

$$
W(\gamma)=E_{\mathbb{P}}\left[(V(\gamma)-\mathbb{X})^{+} \cdot \mathbf{1}_{I}+\mathbb{X}\right]
$$

The conclusion just drawn is based upon the uncertainty parameter $p$ being exogenously given. Our next step is to consider endogenizing this parameter: we enable the manager to select $p$ so as to maximize the ex-ante total value of the firm. This means that the Dye cutoff is now a function of $p$. We thus write $\gamma=\gamma(p)$. It will also be convenient to replace the information-endowment option value $U(\gamma(p))$, as defined in (2.11), by the notation

$$
\Delta(p)=p(\mu-\gamma(p))
$$

for the Dye Disclosure option value which now emphasizes dependence on $p$, that is on the uncertainty of value discovery. 
There are now two possible scenarios with regard to optimal selection of $p$.

In the first scenario (the first-best scenario) it is the manager who is capable of selecting $p$ at no cost, and so is thus able to bargain with the investor for an appropriation of some part $(1-\alpha)$ of the Dye-option value, as in (2.7). In this case his objective is to maximize $\Delta(p)$ over $p$. We show that there is a unique optimizing value $p=\hat{p}$.

Theorem 4 (Uniqueness Theorem). The option value $\Delta(p)$ regarded as a function of $p$ is strictly concave, with a unique maximum located at $p=\hat{p}$, and has the property that $\Delta^{\prime}(0)=\mu$, so that

$$
\max \left\{\Delta^{\prime}(p): p<\hat{p}\right\}=\mu .
$$

For a proof see Section 3.3. We show in Figure 2 the benchmark case of the uniform distribution. It is important to realize that the result of Theorem 4 is likely to be true in any reasonable setting for the market mechanism that might be selected for facilitating informed trading after the interim date. The option value will be zero at the end-points and is likely to be concave on a more general argument based on lottery considerations (see Section 3.4). The unique internal optimum would then still be guaranteed to exist, but explicit forms might well be harder to achieve. This is ultimately our justification here for the simple form (2.6).

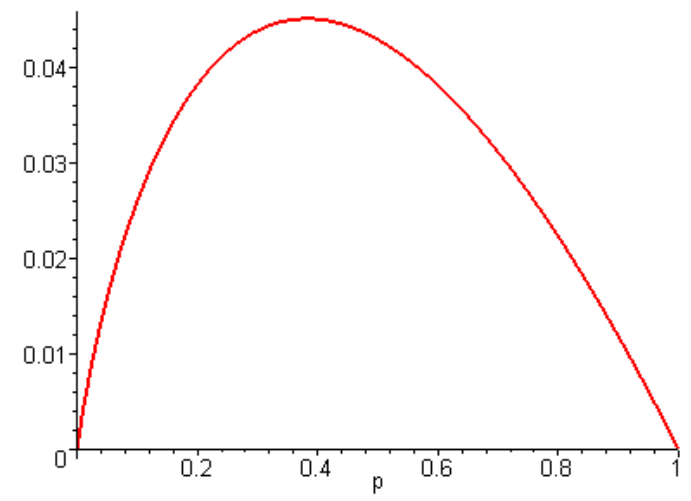

Figure 2. Disclosure-option value $\Delta(p)$ versus uncertainty $p-$ the uniform case.

In the second scenario for the optimal selection of $p$ there is a cost to the manager of acquiring the information endowment and the cost is borne by the firm (since the endowment is regarded as creating additional value for the firm). Now, by Theorem $4, \Delta(p)$ is increasing with $p$ for $p<\hat{p}$, it is thus natural in a first model to assume an acquisition cost proportional to $p$, say of the form $\pi p$, where $\pi$ is an observed constant. Here the manager is rewarded by a share in the residual value of the firm, and hence the manager's objective is to maximize the concave function

$$
\Delta(p)-\pi p
$$

over $p \leq \hat{p}$. The optimizing level $p=\hat{p}(\pi)$ is evidently unique. (The notation is meant to subsume the first scenario as the extreme case in which $\pi=0$, so that $\hat{p}(0)=\hat{p}$.) 


\subsection{Valuing the Dye disclosure option: an example}

In the first-best scenario benchmark case when value is distributed uniformly over $[0,1]$, the maximized option value occurs with $\hat{p}$ at the (complementary) golden mean, which we take to signify $1-\phi=(3-\sqrt{5}) / 2$, or about .38 . The Dye cutoff in this case is also equal to $1-\phi$. The disclosure option is then worth approximately up to a valuable $8 \%$ of ex-ante expected value ${ }^{4}$. Without the acquisition of any additional information, prior to the interim reporting date the expected value of the firm is $\mu=0.5$ (i.e. half of the maximum firm value which we normalize to unity), and, in the case when the manager's contingent claim from the optimized disclosureendowment option is included in the portfolio of an investor, this increases the ex-ante value to 0.545 (as computed in Section 3.2).

Before continuing with the formal analysis, consider briefly the general intuition for the above type of numerical result. A simple implication of the above results is that an optimally imperfectly endowed manager increases the ex-ante total value of the company as compared to the same company without the disclosure option (i.e. a company with a completely unendowed manager) and this evidently depends also on the selected value for $\alpha$ in (2.7). This could and should be construed as ex-ante value creation, by way of utilizing a randomized (mixed) strategy whereby a player (the manager) selects his/her own 'information type' by selecting a probability distribution over the type space $\Omega_{\text {info }}$. This is just one game-theoretic aspect of our analysis: the value of a game can often only be extracted by use of randomized mechanisms rather than pure strategies (akin to the tactic which is sometimes referred to as 'stirring up the fog'). Indeed, we should regard the two scenarios: company with unendowed management, and company with partially endowed management, as respectively a game with informational structure modelled by $\Omega_{\text {value }}$, and a larger game obtained by the addition of informational structure modelled by $\Omega_{\text {info }} \times \Omega_{\text {value }}$. The larger game has larger value. This is the second well-known game-theoretic aspect brought out by our analysis: if you don't like the game you're in, then embed the game into a 'larger game'. On both matters refer to Brandenburger and Nalebuff (1996).

Of course this comes at risk: at the interim reporting date the value of the larger game could drop to $V(t)$, as compared to being maintained at $\mu$ in the smaller game. The larger game benefits an investor wishing to sell at the ex-ante date by way of the total value.

\subsection{Qualitative properties}

Having identified that a unique optimizing $p$ exists (which implies a unique Dye option value), it is next natural to ask what general qualitative properties can be determined for the optimal Dye disclosure option. Below we formally show:

(a) that it is never optimal for the manager to become fully informed $(\hat{p} \neq 0)$, and we explain how this follows intuitively from the presence of insurance cover provided from the protective put (see Proposition 4 of Section 3.3);

(b) that it is never optimal for the manager's odds ${ }^{5}$ against being informed to be greater than 1. That is, in the optimal situation $\hat{q}=1-\hat{p}>\hat{p}$. This follows from tracking the consequence of the uncertainty parameter $p$ when decreasing it infinitesimally from the optimum. There needs to be a trade off between the infinitesimal gain in being informed slightly more frequently and the infinitesimal loss in the reduced shielding from 'bad news' (previously hidden by non-disclosure). More precisely, the increase in the unconditional probability $q$ (probability of the manager being

\footnotetext{
${ }^{4}$ Strictly speaking, this is value per share permitted to be traded by the informed manager.

${ }^{5}$ Recall that the 'odds against' this event are given by the ratio $p / q$; this ratio will be denoted $\lambda$.
} 
informed) equals the drop in the conditional probability of an informed manager not disclosing. (Again see Proposition 4 of Section 3.3).

In a companion paper we have investigated the dependence of the disclosure option value both on $p$ and on the distribution $F$ and have derived comparative statics of various model parameters, such as $\gamma(p)$. We have also found upper and lower bounds on both $\gamma(p)$ as well as on $\hat{p}$, the 'optimal informational-uncertainty'; in the case of $\gamma(p)$ for a general distribution $F$ the bounds refer to the variance $p(1-p)$ and to some other natural risk measures. As a sample, we quote a crude estimate of the 'disclosure option' value which takes the form

$$
p(1-p) E_{F}[(\mu-X), X<\mu] .
$$

This simple formula identifies the essential nature of the option value in that it depends on the binomial variance effect, $p(1-p)$, and quite naturally on the expectation of low value discovery, that is on 'below mean-target performance', in the words of Fishburn (1977).

(c) Finally, we turn to an intuitive interpretation of the expectations that arise in characterizing and valuing the Dye cutoff. We show that many of the considerations may be reduced to working with the Omega function which arises in portfolio risk management, i.e. the gain-to-loss ratio relative to a target $t$.

Let us consider how the disclosure cutoff $t$ categorizes risk. The downside risk is this: when the realized value $x$ has $x<t$, the notional loss in share value is $(t-x)$ relative to a full disclosure. The non-disclosure protects the investor from this loss, giving the protective shield a value, namely the expected 'notional gain', which equals

$$
\int_{0}^{t}(t-x) f(x) d x .
$$

This gain is offset simultaneously by the upside risk. Suppose now that the realized value $x$ has $x>t$. A manager, if informed of $x$, will report $x>t$, and the investors will have a net gain of $(x-t)$. No such gain arises if the manager is uninformed. Thus the expected 'foregone notional gain' resulting from imperfect information is

$$
p \int_{t}^{1}(x-t) f(x) d x .
$$

At equilibrium the gain must equal the loss. That is,

$$
p \int_{t}^{1}(x-t) f(x) d x=\int_{0}^{t}(t-x) f(x) d x,
$$

or

$$
\frac{\int_{t}^{1}(x-t) f(x) d x}{\int_{0}^{t}(t-x) f(x) d x}=\frac{1}{p} .
$$

The 'gain-to-loss ratio' on the left-hand side may be re-written in the equivalent form

$$
\Omega(t)=\frac{\int_{t}^{1}(1-F(x)) d x}{\int_{0}^{t} F(x) d x}=\frac{1}{p},
$$

in view of the following two identities (verifiable e.g. by integration by parts) namely:

$$
\int_{t}^{1}(x-t) f(x) d x=\int_{t}^{1}(1-F(x)) d x, \quad \int_{0}^{t}(t-x) f(x) d x=\int_{0}^{t} F(x) d x .
$$


The notation is due to Keating and Shadwick (2002a). To summarize: with reference to (2.14) we have shown the following.

Proposition. Optimal risk-shielding via the Dye disclosure strategy is characterized by the cutoff level $t$ satisfying the equation

$$
p \Omega(t)=1
$$

Let us concentrate on the left-hand side expression in the later of the identities of (2.16). This integral has the structure of a put valuation $P_{t}$. Its dependence on $t$ is crucial to our considerations (the earlier identity has of course a call valuation structure). We propose the term 'hemi-mean' function, as defined and denoted by:

$$
H(t)=\int_{0}^{t}(t-x) f(x) d x .
$$

This function represents an expected shortfall relative to $t$, and is also known as the first lower partial moment, $\mathrm{LPM}_{1}$ and is also called the 'target shortfall' in Harlow (1991). See also Bawa (1975). Our chosen terminology is by analogy to the lower 'semi-variance' which refers to $\mathrm{LPM}_{2}$, see McNeil, Frey and Embrechts (2005). It is our thesis that the behaviour of the cutoff $t$ is dictated only by (distributional) properties of $F(x)$ but only in respect of the lower mean interval $[0, \mu]$, so, more properly, on tghe properties of the hemi-mean.

After some manipulation, starting from the put-call parity property

$$
\int_{t}^{1}(x-t) f(x) d x=(\mu-t)+H(t)
$$

(deducible from the facts that $\int_{0}^{1} f(x) d x=1$ and $\int_{0}^{1} x f(x) d x=\mu$ ), one may reformulate $(2.17$ ) to the form

$$
\frac{H(t)}{\mu-t}=\frac{p}{q}
$$

as given in Jung and Kwon (1988), albeit in our current notation.

The appearance of the $\Omega$-statistic should not come as a complete surprise - it has recently been discussed in the portfolio risk-management literature on target $(t)$ returns, since its introduction in Keating and Shadwick (2002a). Its salient mathematical features are given in Cascon, A., Keating, C., Shadwick, W.F.(2002).

In our investigations the most convenient primitive tool is the $H(t)$ function, and equation (2.18) is just one justification. It is a natural tool especially if one reflects on the perfectly informed, fully disclosing manager who reports $x<\mu$; here the investor suffers a loss of $\mu-x$ resulting from the disclosure (relative to the ex-ante valuation $\mu$ ). Accordingly, a relevant statistic for the investor is the lower partial moment $E_{F}[\mu-X, X<\mu]=H(\mu)$, cf. (2.13).

Evidently,

$$
\Omega(t)=\Lambda(t)+1, \text { where } \Lambda(t)=\frac{\mu-t}{H(t)}
$$

and which of these functions, $\Omega$ or $\Lambda$, to use for analysis very much depends on context. For example, in studying sensitivity to stochastic dominance, if $F_{1}$ dominates $F_{2}$ (in second order, or a fortiori, in first order), then clearly $\Lambda_{1}$ majorizes $\Lambda_{2}$, in which case $\Lambda(t)$ is increasing in $t$, it is obvious that $\gamma_{1}>\gamma_{2}$ (for fixed $p$ ). 
The generalized risk measure of Bernell Stone (1973) over distributions $F$ is defined by reference to deviations below a range cutoff $\gamma(F)$, from a target level $\eta(F)$, by the formula

$$
\rho_{\alpha}(F)=\int_{0}^{\gamma(F)}|\eta(F)-x|^{\alpha} d F(x) .
$$

Thus $H(\mu)$, the hemi-mean at $\mu$, is merely $\rho_{1}(F)$ with $\gamma(F)$ taken to be $\mu$. Evidently $F(\mu)=\rho_{0}(F)$. But estimates refining (2.13) naturally lead to the exogenous range cutoff $\gamma(p)$, and the endogenous range cutoff $\gamma(\hat{p})$. We explore these notions and the appropriate concepts of stochastic dominance in a companion paper.

\section{Further properties of the optimal cutoff}

In this section some fundamental properties of the Dye cutoff will be derived. First of all a derivation of the explicit form of $V(t)$ will be presented, and then applied to the case of a specific distributional assumption to offer some simple benchmark results.

\subsection{Some explicit formulas}

We recall the explicit formula for $V(t)$, which we will also write as $V(t, p)$ whenever the choice of $p$ needs to be clear. We will use the notation

$$
F(t)=\int_{0}^{t} f(x) d x, \quad E(t)=\int_{0}^{t} x f(x) d x,
$$

and we recall that $\mu=E_{F}[X]$, so that $0<\mu<1$. With this notation, we note that the hemi-mean satisfies

$$
H(t)=t F(t)-E(t)
$$

If the strike value $t$ is known, then, conditional on no disclosure, which is an event of probability $p+q F(t)$, the risk-neutral investors compute the conditional expected value of the firm to be $V(t)$, where:

$$
V(t)=V(t, p)=\frac{p \mu+q E(t)}{p+q F(t)}
$$

(as in Jung and Kwon, (1988)), which is the explicit version of (2.1). This is a continuous function of $t$. Since $0<\mu<1$ and $V(0)=\mu>0$ and $V(1)=\mu<1$, by continuity, there is at least one value $t$ such that $V(t)=t$. The equation $V(t)=t$ may be rewritten in the equivalent implicit relation:

$$
Y(t, p)=p(\mu-t)-q(t F(t)-E(t))=0 .
$$

The function $Y(., p)$ is an increasing, convex function with $Y(0, p)<0$ and $Y(\mu, p)>0$, so there is a unique $\gamma=\gamma(p)$ such that $Y(\gamma, p)=0$.

\subsection{The benchmark example: the uniform case}

For the uniform distribution $f(x) \equiv 1$, we have $\mu=0.5$ and $F(\mu)=\mu$, and $E(\mu)=\frac{1}{2} \mu^{2}=0.125$.

For a feel of the context, take $p=q=0.5$ to obtain

$$
V(t)=\frac{1+t^{2}}{2(1+t)}
$$


so that $\gamma$ is the positive root of $t^{2}+2 t-1=0$, i.e. $\gamma=\sqrt{2}-1=0.414$. The option value of disclosure is thus $\Delta(0.5)=0.5(0.5-0.414)=0.043$.

For general $p$, we have

$$
\gamma(p)=\frac{\sqrt{p}}{1+\sqrt{p}}, \quad \Delta(p)=\frac{p(1-\sqrt{p})}{2(1+\sqrt{p})}
$$

The Dye cutoff and the disclosure-option value as functions of the uncertainty $p$ are illustrated respectively in Figure 2 and Figure 3.

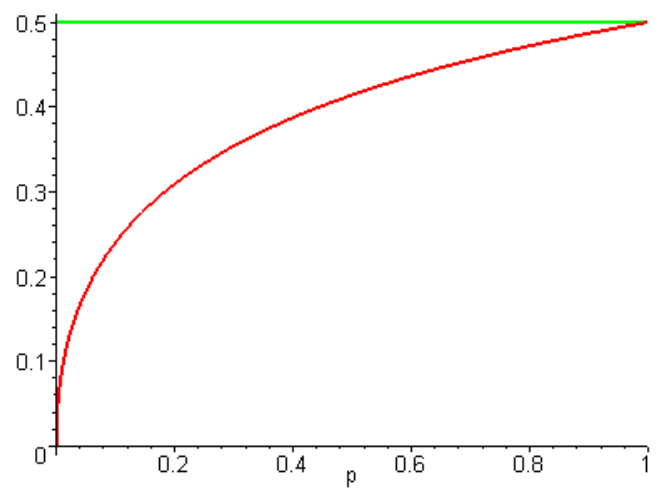

Figure 3. Dye cutoff $\gamma(p)$ versus uncertainty $p$ - the uniform case.

By routine calculus, the option value $\Delta(p)$ is maximized at

$$
p=\hat{p}=\frac{1}{2}(3-\sqrt{5}) \approx .381966011,
$$

with $\gamma(\hat{p})=0.381966$. The option value is worth

$$
\Delta(\hat{p})=\frac{1}{4}(5 \sqrt{5}-11) \approx .045084972 .
$$

The option-value as a contribution of total pre-discovery expected firm value is thus over $8.25 \%$.

\subsection{Fundamental properties}

Jung and Kwon showed that $\gamma(p)$ is strictly increasing in $p$. This entitles us to consider the equivalent inverse relation $p=p(g)$, whereby we regard the cutoff value $g$ as the independent variable ranging over $[0, \mu]$, while the originating uncertainty $p$ becomes the dependent variable (it is then the 'implied uncertainty parameter'). We will switch back and forth between equivalent formulations according to need, bearing in mind that we wish to continue working with explicit formulas. Thus when the Dye option value $\Delta(p)$ is to be equivalently viewed as a function of the cutoff $g$ we will write the option value as $\delta(g)$.

Proposition 1. The uncertainty of discovery, $p$, as a function of the Dye cutoff $g$, is given, for $0 \leq g \leq \mu$, by

$$
p=p(g)=\frac{H(g)}{(\mu-g)+H(g)}
$$


It is an increasing, convex function with extreme values $p(0)=0$ and $p(\mu)=1$, and with extreme slopes $p^{\prime}(0)=0$ and $p^{\prime}(\mu)=1 / H(\mu)$.

We illustrate this result in Figure 4 for the uniform case.

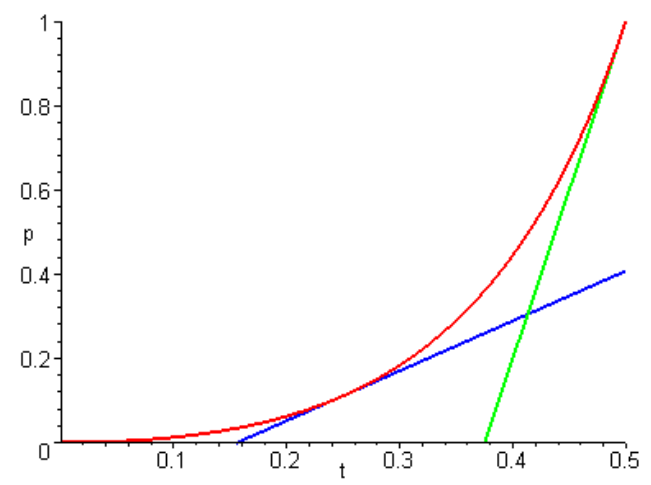

Figure 4. $p(g)$ for $0 \leq g \leq \mu$ in the uniform case.

Proof. The defining equation for the cutoff $\gamma=\gamma(p)$ is $V(t)=t$, which in explicit terms is

$$
g=V(g, p)=\frac{p \mu+q E(g)}{p+q F(g)}
$$

or

$$
p(\mu-g)=q(g F(g)-E(g))=q H(g) .
$$

This may be solved for $p$ as asserted. From (3.3) we compute that

$$
\frac{d p}{d g}=\frac{F(g)(\mu-g)+H(g)}{((\mu-g)+H(g))^{2}},
$$

which is positive for $g<\mu$. Furthermore,

$$
\frac{d^{2} p}{d g^{2}}=\frac{[f(g)(\mu-g)]((\mu-g)+H(g))^{2}+[F(g)(\mu-g)+H(g)][1-F(g)]}{((\mu-g)+H(g))^{4}},
$$

which is seen to be positive for $g<\mu$.

Proposition 2 (Marginal Trade-off). The marginal value of the Dye cutoff is related to the disclosure option value as follows:

$$
p q d \gamma=\Delta(p) \frac{d p}{\mathbb{P}(N(\gamma))}
$$

Proof. The substitution $H(g)=\lambda(\mu-g)$, with $\lambda=\frac{p}{q}$ denoting the odds in (3.4), and, noting that $1+\lambda=\frac{1}{q}$, leads for $g=\gamma(p)$ to

$$
\frac{d \gamma}{d p}=\frac{((\mu-\gamma)+H(\gamma))^{2}}{F(\gamma)(\mu-\gamma)+H(\gamma)}=\frac{(\mu-\gamma)^{2}(1+\lambda)^{2}}{(\mu-\gamma)[F(\gamma)+\lambda]}=\frac{p(\mu-\gamma)}{p q(p+q F(\gamma))}
$$


That is,

$$
\frac{d \gamma}{d p}=\frac{\Delta(p)}{p q(p+q F(g))}
$$

Comments. The formula (3.5) may be interpreted as a trade-off between a loss of value in $W$ and a gain in option-value $\Delta$. As the cutoff change $d \gamma$ upgrades or downgrades valuation in the set $N(\gamma)$, the incremental loss or gain in $W$ is $d W \mathbb{P}(N(\gamma))$, which is equal by (8.2) to $p q\left(1-V^{\prime}(\gamma)\right) d \gamma$, and this simplifies to the variance effect $p q d \gamma$, since $V^{\prime}(\gamma)=0$ (by our characterization of the Dye cutoff). The right-hand side of formula (3.5) is the corresponding incremental gain or loss in option-value obtained from multiplying the increment in probability, equal to $d p / \mathbb{P}(N(\gamma))$, by the current option value $\Delta(p)$.

The fact that $d \gamma / d p>0$ was first shown in Jung and Kwon (1988) by implicit differentiation of $Y(t, p)$. Here we have explained their result by interpreting the marginal substitution effects as trade-offs.

Proposition 3. The value of the disclosure option, as a function of the optimal cutoff, is given by

$$
\delta=\delta(g)=\frac{(\mu-g) H(g)}{H(g)+(\mu-g)} .
$$

It is strictly increasing in the range $[0, \mu / 2]$, hence is maximized in the range $[\mu / 2, \mu]$. The derivative satisfies

$$
\frac{d \delta}{d g}=q(g)^{2}\left(F(g)-\left(\frac{p(g)}{1-p(g)}\right)^{2}\right) .
$$

Proof. The defining equation for the option value is $\delta=p(\mu-g)$, hence the formula follows from (3.3) of Proposition 1. We may now compute that

$$
\delta^{\prime}(g)=\frac{d \delta}{d g}=\frac{(\mu-g)^{2} F(g)-H^{2}(g)}{[H(g)+(\mu-g)]^{2}} .
$$

Hence we have

$$
\delta^{\prime}(0)=0, \quad \delta^{\prime}(\mu)=-1<0 .
$$

Moreover, for $0<g \leq \mu / 2$, we have $g \leq \mu-g$, so, since $F<1$,

$$
(\mu-g)^{2} F(g)-H^{2}(g) \geq g^{2} F(g)-H^{2}(g)>g^{2} F^{2}(g)-H^{2}(g)>0 .
$$

Thus $\delta$ has a stationary point in $(\mu / 2, \mu)$.We will see in Theorem 4 below that this location is unique.

The last assertion of the theorem follows on using the substitution $H(g)=\lambda(\mu-g)$, where $\lambda=\frac{p}{q}$ in (3.7). Indeed, we have

$$
\frac{d \delta}{d g}=\frac{F(g)-\lambda^{2}}{(\lambda+1)^{2}}=q^{2}\left(F(g)-\left(\frac{p}{q}\right)^{2}\right) .
$$

Proposition 4 (Optimal uncertainty). Stationarity of $\delta(g)$ occurs in the open interval $0<$ $p<1$ iff $g=\gamma(p)$ and

$$
\sqrt{F(g)}=\frac{p}{1-p}
$$


and hence

$$
0<p<\frac{1}{2}
$$

The optimality condition for $p$ may be restated as

$$
q=1-p=\frac{p}{p+q F(\gamma(p))}=\frac{p}{\mathbb{P}[N(\gamma)]}
$$

Comment. The equation (3.8) is useful for tracking marginal trade-offs. Suppose that the uncertainty parameter is decreased infinitesimally. The equation (3.8) then asserts a trade-off between the infinitesimal gain from being informed with an infinitesimal loss of the probability of shielding from bad news by non-disclosure.

Proof. Since $F(\gamma)<F(\mu)$, we have, at the optimal $p$, that in fact

$$
p=\frac{\sqrt{F(\gamma)}}{1+\sqrt{F(\gamma)}}<\frac{\sqrt{F(\mu)}}{1+\sqrt{F(\mu)}}<\frac{1}{2} .
$$

As regards the restatement of the optimality condition, note that the condition $F(\gamma)=p^{2} / q^{2}$ is equivalent to

$$
\frac{p}{q}+F(\gamma)=\frac{p}{q}\left(1+\frac{p}{q}\right)=\frac{p}{q^{2}}
$$

The latter may be rearranged to read :

$$
p+q F(\gamma)=\frac{p}{q}
$$

from which the result (3.8) follows.

Theorem 4 (Uniqueness Theorem). The option value, regarded as a function of $p$, is strictly concave with $\Delta^{\prime}(0)=\mu$ and $\Delta^{\prime}(1)=-H(\mu)$, so that its maximum $\hat{p}$ is unique and

$$
\max \left\{\Delta^{\prime}(p): p<\hat{p}\right\}=\mu .
$$

See Section 3.4 for an informal game-theoretic proof of concavity.

Proof. Recalling that $\Delta(p)=p(\mu-\gamma(p))$, we have

$$
\frac{d \Delta}{d p}=(\mu-\gamma(p))-p \frac{d \gamma}{d p}
$$

Notice that, for $p=1$, we have $\gamma(1)=\mu$, so referring to the formula for $d p / d \gamma$, as in (3.4), we have

$$
\Delta^{\prime}(1)=-H(\mu) .
$$

Next we observe that

$$
\frac{1}{p} \frac{d p}{d \gamma}=\frac{F(\gamma)(\mu-\gamma)+H(\gamma)}{H(\gamma)[(\mu-\gamma)+H(\gamma)]}=\frac{1+\frac{F(\gamma)}{H(\gamma)}(\mu-\gamma)}{(\mu-\gamma)+H(\gamma)}
$$

But

$$
\frac{F(\gamma)}{H(\gamma)} \geq \frac{1}{\gamma}
$$


so our result concerning the extreme slope follows from the observation that, by (3.11), we have

$$
\lim _{p \rightarrow 0} \frac{1}{p} \frac{d p}{d \gamma}=+\infty
$$

Now, recalling the formula (3.6)

$$
\frac{d \gamma}{d p}=\frac{\Delta(p)}{p q(p+q F(\gamma))}
$$

and referring to (3.10) above, we have, by the quotient rule of calculus, that

$$
\begin{aligned}
\frac{d^{2} \gamma}{d p^{2}} & =\frac{\left[(\mu-\gamma(p))-p \frac{d \gamma}{d p}\right] p q(p+q F(\gamma))-e t c}{[p q(p+q F(\gamma))]^{2}} \\
& =\frac{\left[\frac{\Delta(p)}{p}-p \frac{\Delta(p)}{p q(p+q F(\gamma))}\right] p q(p+q F(\gamma))-e t c}{[p q(p+q F(\gamma))]^{2}} \\
& =\frac{\Delta(p)(q(p+q F(\gamma))-p)-\Delta(p)\left[q(p+q F(\gamma))-p(p+q F(\gamma))+p q\left(1-F(\gamma)+\frac{f \Delta(p)}{p(p+q F(\gamma))}\right)\right]}{[p q(p+q F(\gamma))]^{2}} \\
& =-\Delta(p) \frac{p[1-(p+q F(\gamma))]+p q\left([1-F(\gamma)]+\frac{f \Delta(p)}{p(p+q F(\gamma))}\right)}{[p q(p+q F(\gamma))]^{2}}<0 .
\end{aligned}
$$

Here $f=f(\gamma)=F^{\prime}(\gamma)$.

Corollary. If a price $\pi p$ with $\pi>0$ were to be paid for purchasing the option with uncertainty $p$, then

i) the option is worth buying if and only if the price $\pi$ per unit of uncertainty satisfies $\pi<\mu$;

ii) if the option is worth buying, then the corresponding unique optimal level of uncertainty $p=$ $p(\pi)$ satisfies $p(\pi)<\hat{p}$

iii) the value of the option decreases strictly with $\pi$, for $\pi<\mu$.

Proof. If a price $\pi$ were to be paid per unit of uncertainty for purchasing the option, then the optimal level $p=\hat{p}(\pi)$ must maximize the concave function

$$
\Delta(p)-p \pi
$$

and hence solves the equation

$$
\pi=\Delta^{\prime}(\hat{p}(\pi))
$$

Hence $\pi<\max \left\{\Delta^{\prime}(p): p<\hat{p}\right\}<\mu$, and moreover, with $p=\hat{p}(\pi)$, we have

$$
\pi<p(\mu-\gamma(p))
$$

so that again

$$
\pi<\mu-\gamma(p)<\mu \text {. }
$$

Now, as $0<\pi<\mu=\max \left\{\Delta^{\prime}(p): p<\hat{p}\right\}$, there is indeed a unique solution $p=\hat{p}(\pi)$ to the first-order condition $\pi=\Delta^{\prime}(p)$. Since we have $\Delta^{\prime}(p)<0$, for $p>\hat{p}$, the solution $p(\pi)$ satisfies

$$
\hat{p}(\pi)<\hat{p} .
$$


It remains to prove that

$$
\hat{\Delta}(\pi):=\Delta(\hat{p}(\pi))-\pi \hat{p}(\pi)
$$

is positive. Note that $\hat{\Delta}(\pi)$ is convex, being the negative of the Fenchel dual ${ }^{6}$ of $\Delta$. Indeed, as in the Envelope Theorem,

$$
\begin{aligned}
\frac{d \hat{\Delta}}{d \pi} & =\Delta^{\prime}(\hat{p}(\pi)) \frac{d \hat{p}}{d \pi}-\hat{p}(\pi)-\pi \frac{d \hat{p}}{d \pi} \\
& =-\hat{p}(\pi),
\end{aligned}
$$

since $\pi=\Delta^{\prime}(p(\pi))$. Thus $\hat{\Delta}(\pi)$ is strictly decreasing in $\pi$ for $0<\pi<\mu$. But for $\pi=\mu$ we obtain $\hat{p}(\pi)=\hat{p}(\mu)=0$, and so $\hat{\Delta}(\mu)=0$. Hence $\hat{\Delta}(\pi)>0$ for $\pi<\mu$. In particular of course

$$
\pi p(\pi)<\hat{p}(\mu-\gamma(\hat{p}))
$$

\subsection{An informal game-theoretic approach to concavity}

Here is an informal game-theoretic proof of Theorem 4. $\Delta(p)$ is the value of a gamble. Consider two such gambles: for $p=p_{0}$ and for $p=p_{1}$, and further consider the following composite gamble. The initial step is a lottery determining a move to one of the two gambles with manager's value $\Delta\left(p_{i}\right)$. We consider two version of this game. In the first we intend that neither the manager, nor the other market participants, observes the outcome of the initial lottery, but all agents know that the chance move, leading to the subgame with uncertainty parameter $p_{0}$, has probability $1-r$.

In a variant game, assuming all players observe the outcome of the composite game, the manager computes the value, given the additionally available information to be

$$
(1-r) \Delta\left(p_{0}\right)+r \Delta\left(p_{1}\right)
$$

However, the probabilities $p_{i}$ identify the uncertainty of being informed and, without needing to observe the outcome of the first stage lottery, the manager knows that the probability of being uninformed is

$$
p_{r}=(1-r) p_{0}+r p_{1} .
$$

This is enough information to determine how to behave optimally. Thus the manager might as well be allowed to observe the outcome of the lottery.

What is the connection between

$$
\Delta\left(p_{r}\right)=\mu+p_{r}\left(\mu-\gamma\left(p_{r}\right)\right)
$$

and

$$
r \Delta\left(p_{0}\right)+(1-r) \Delta\left(p_{1}\right) ?
$$

To answer this question, consider the uninformed market participants. If they observe the first step lottery, they know which of the two subgames the market moves to, and therefore what valuation calculation to make. If they do not observe the initial lottery, they are at a disadvantage, and so

\footnotetext{
${ }^{6}$ Formally, the Fenchel dual of the concave function $\Delta(p)$ is the concave function of $\pi$ given by

$$
\Delta^{*}(\pi)=\inf _{p}[p \pi-\Delta(p)]
$$
}


the manager has an advantage. The value of the game in which only the manager observes the initial lottery is thus surely greater to the manager. Hence

$$
\Delta\left(p_{r}\right)>(1-r) \Delta\left(p_{0}\right)+r \Delta\left(p_{1}\right)
$$

for $0<r<1$. This proves concavity.

\section{Disclosure intensity}

Proposition. The intensity of disclosure, that is the ex-ante risk-neutral probability of a disclosure occurring, defined by

$$
\tau(p)=(1-p)(1-F(\gamma(p)))
$$

is a decreasing function of $p$. The extreme values are $\tau(0)=1$ and $\tau(1)=0$, and the extreme slopes are $\tau^{\prime}(0)=-\infty$ and $\tau^{\prime}(1)=-(1-\mu)=-H(1)$.

If it is a convex function of $p$, which is the case if for instance $f^{\prime}(t) \leq 0$ in the region $(0, \mu)$, then

$$
\tau(p) \geq(1-\mu)(1-p) .
$$

We illustrate the result in Figure 5 for the uniform case.

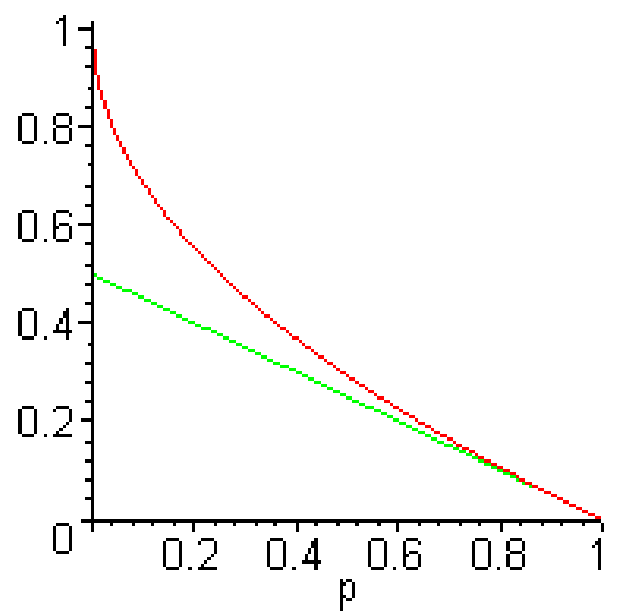

Figure 5. Disclosure intensity $\tau(p)$ against $p$ (bold) and tangential approximation (faint) - in the uniform case.

Proof. The monotonicity result is obvious, since both $1-p$ and $1-F(\gamma(p))$ are decreasing with $p$ (the latter factor because both $F(t)$ and $\gamma(t)$ are increasing with $t$ ). Since $d \gamma / d p>0$ and $d^{2} p / d \gamma^{2}>0$ (by Proposition 1), routine calculations starting from

$$
\tau^{\prime}(p)=-\left((1-\gamma)+(1-p) f(\gamma) \frac{d \gamma}{d p}\right)<0
$$

lead to

$$
\tau^{\prime \prime}(p)=(1+f(\gamma)) \frac{d \gamma}{d p}-(1-p) f^{\prime}(\gamma)\left(\frac{d \gamma}{d p}\right)^{2}+(1-p) f(\gamma) \frac{d^{2} p}{d g^{2}}\left(\frac{d \gamma}{d p}\right)^{3}>0
$$


Proposition. It is the case that

$$
\tau(\hat{p}) \geq \hat{p} \text { iff } \hat{p} \leq \frac{1}{2}(3-\sqrt{5})=.38 \ldots .
$$

Proof. We have, since $F(\gamma(\hat{p}))=\lambda^{2}$,

$$
\tau(\hat{p})=(1-\hat{p})\left(1-F(\gamma(\hat{p}))=\frac{1-\lambda^{2}}{1+\lambda}=1-\lambda .\right.
$$

We may now argue that

$$
1-\lambda \geq \hat{p}
$$

is equivalent to

$$
1-\hat{p} \geq \lambda=\frac{\hat{p}}{1-\hat{p}}
$$

that is to

$$
1-3 \hat{p}+\hat{p}^{2} \geq 0
$$

from which our result follows.

\section{Densities with an initial power form}

Suppose it is the case, for $0 \leq t \leq \mu$, that $f(t)=K t^{\alpha-1}$, with $\alpha>0$. We call such densities 'initially of index $\alpha$ '.

Proposition. If the density is initially of index $\alpha$ then the Dye cutoff $\gamma(\hat{p})$ satisfies

$$
\frac{\alpha}{\alpha+2} \mu<\gamma(\hat{p})<\mu \text {. }
$$

Proof. Recall that the optimality condition satisfied by $p=\hat{p}(\gamma)$ takes the form

$$
F(g(p))(\mu-g)^{2}=H(g)^{2} .
$$

We note that $F(t)=K t^{\alpha} / \alpha$ and $H(t)=K t^{\alpha+1} / \alpha(\alpha+1)$. Observe that, since $F(\mu) \leq 1$, we also have

$$
\frac{K}{\alpha} \mu^{\alpha} \leq 1 .
$$

We show that the maximum value of $F(t)(\mu-t)^{2}$ in $(0, \mu)$ occurs at $t=\theta \mu$, where

$$
\theta=\frac{\alpha}{\alpha+2},
$$

and that $F(\theta \mu)(\mu-\theta \mu)^{2}>H(\theta \mu)^{2}$. It follows that the optimality condition is satisfied uniquely in $[\theta \mu, \mu]$.

Step 1. We find the location of the maximum of the function $F(t)(\mu-t)^{2}$ in $(0, \mu)$.

Solving

$$
0=f(t)(\mu-t)^{2}-2 F(t)(\mu-t)=K(\mu-t) t^{\alpha-1}\left[(\mu-t)-\frac{2}{\alpha} t\right]
$$

yields $t=\theta \mu$, as asserted. 
Step 2 We note that the maximum value is as follows:

$$
M=\frac{K}{\alpha}(\theta \mu)^{\alpha} \mu^{2}(1-\theta)^{2}=\frac{K}{\alpha} \mu^{\alpha+2}\left(1+\frac{2}{\alpha}\right)^{-\alpha}\left(\frac{2}{\alpha+2}\right)^{2} .
$$

Step 3. This maximum $M$ is above $H(\theta \mu)^{2}$ iff

$$
4\left(1+\frac{2}{\alpha}\right)^{\alpha}\left(\frac{\alpha+1}{\alpha+2}\right)^{2} \geq \frac{K}{\alpha} \mu^{\alpha} .
$$

But, for $\alpha>0$, we have

$$
\frac{\alpha+1}{\alpha+2} \geq \frac{1}{2}, \quad \text { and } \quad\left(1+\frac{2}{\alpha}\right)^{\alpha}>1 .
$$

Thus we have, for $\alpha>0$, that

$$
4\left(1+\frac{2}{\alpha}\right)^{\alpha}\left(\frac{\alpha+1}{\alpha+2}\right)^{2}>1 \geq \frac{K}{\alpha} \mu^{\alpha},
$$

and so the function $H(t)^{2}$ intersects $F(t)(\mu-t)^{2}$ in the interval $\left(\frac{\alpha \mu}{\alpha+2}, \mu\right)$.

Notice that

$$
\lim _{\alpha \rightarrow \infty}\left[4\left(1+\frac{2}{\alpha}\right)^{\alpha}\left(\frac{\alpha+1}{\alpha+2}\right)^{2}\right]=4 e^{2}=29.55 \ldots,
$$

so the maximum $M$ is liable to be significantly above $H(\theta \mu)^{2}$.

\section{Conclusion}

We have refined the original Dye disclosure model by recognizing that the possibility of an upper tailed disclosure strategy confers an option on the manager, namely whether to disclose or not to disclose firm value above a selected cutoff. Dye's motivation for the lack of credible communication, when the manager is uninformed, was principally to show why unravelling does not necessarily happen. While lack of unravelling clearly had implications for existing investors, this was not his principle concern at that time when he established his possibility theorem for a cutoff disclosure strategy. In our research we concentrate on these value implications, while preserving the same qualitative cutoff disclosure features of the Dye model. Once one becomes concerned about the value implications to investors of a Dye disclosure strategy, one becomes sensitive to the trading assumptions that managers are allowed to operate with. We show that in the event of non-disclosure investors will be less harmed if management are allowed to trade when they know that they do not know firm value. Said another way: we show how investors can benefit (receive additional value) when the market devalues the company, following non-disclosure, yet management know this non-disclosure arises from no new information being received (rather than from discovery of low value).

The value of the 'Dye option' depends on both the location of the cutoff and on the probability $q$ of the manager being perfectly informed as to firm value. Given the probability $q$, the option value is found to be maximized at the cutoff level originally identified by Dye.

The Dye cutoff level is a function of the probability $q$. The maximized option value is correspondingly also a function of the probability $q$, and in its dependence on this variable it too may 
be maximized by a manager empowered to select the value of $q$. Thus one of the contributions of this paper is to provide a formal link between the manager's own choice of information type and ex-ante additional value creation. Impounding this 'additional value' into a total value of the firm, and using our contingent claims analysis with some representative parameter values, allows us to demonstrate that the Dye disclosure option can add up to something like $8 \%$ per share to the value of the firm. Furthermore we provide a new link between corporate disclosure strategy and risk-management methodology. The link we identify refers to the risk-measure known as the Omega function, which takes the form of a gain-to-loss ratio. We show how the Dye cutoff level corresponds to this gain-to-loss ratio being chosen so as to provide optimal risk-shielding to the investor. 


\section{References}

Baginski, S.P., Hassell, J.M., and Hillison, W.A., "Voluntary causal disclosures:tendencies and capital market reactions", Review of Quantitative Finance and Accounting, 15, 371-389, (2000)

Baiman, S., and Verrecchia, R.E., "The Relation among Capital Markets, Financial Disclosure, Production Efficiency, and Insider Trading, Journal of Accounting Research , 34.1, 1-22, (1992)

Brandenburger, A., and Nalebuff, B.J., "Co-opetition", Currency Doubleday, (1996)

Bawa, V.S., "Optimal Rules for ordering uncertain prospects", Journal of Financial Economics, 2, 95-121, (1975)

Campbell, K., Sefcik, S.E., and Soderstrom, N.S., "Disclosure of private information and reduction of uncertainty: environmental liabilities in the chemical industry", Review of Quantitative Finance and Accounting, 21, 349-378, (2003)

Cascon, A., Keating, C., and Shadwick, W.F., "The Omega Function", The Finance Development Centre, London, Working paper, (2002)

Christensen, P.O., Feltham, G.A., "Economics of Accounting - I", Kluwer Academic Publishing, (2003)

Cosimano, T.F., Jorgensen, B.N., and Ramanan, R., "Discretionary Disclosure Over Time", Working Paper, (2002)

Dye, R.A., "Disclosure of Nonproprietary Information", Journal of Accounting Research, 23, 123-145, (1985)

Einhorn, E., and Ziv,A., "Inter-temporal Dynamics of Corporate Voluntary Disclosure", Working Paper TAU, (2005a).

Einhorn, E., and Ziv,A., "Intra- and Inter-temporal Dynamics of Corporate Disclosure and Operating Activities", Working Paper TAU, (2005b)

Fishburn, P.C., "Mean-Risk Analysis with Risk Associated with Below-Target Returns", American Econonomic Review, 67,116-126, (1977)

Grossman, S., and Hart, O. "Disclosure Laws and Take-over bids", Journal of Finance, 35, 323-34, (1980)

Grossman, S., "The Informational Role of Warranties and Private Disclosure about Product Quality" Journal of Law and Economics, 24, 461-483, (1981)

Harlow, W., and Rao, R., "Asset pricing in a generalized mean-lower partial moment framework: Theory and evidence, Journal of Financial and Quantitative Analysis, 24, 285-311, (1989).

Harlow, W. V., "Asset Allocation in a Downside-Risk Framework", Financial Analysts Journal, September, 28-40, (1991)

Hughes, J.S., and Pae, S., "Voluntary Disclosure of Precision Information", Journal of Accounting and Economics, 37, 261-289, (2004)

Keating, C., and Shadwick, W.F., "An Introduction to $\Omega$ ", The Finance Development Centre, London, Working paper, (2002a)

Keating, C., and Shadwick, W.F., "A Universal Performance measure", The Finance Development Centre, London, Working paper, (2002b)

Leland, H. E., "Insider Trading: Should It Be Prohibited?", Journal of Political Economy, 100 (December), 859-887, (1992)

McNeil, A.J, Frey, R., and Embrechts, P., "Quantitative Risk management", Princeton University Press, (2005)

Pae, S., "Selective Disclosures in the Presence of Uncertainty about Information Endowment, Journal of Accounting Economics", 39, 383-409, (2005) 
Penno, M.C., "Information Quality and Voluntary Disclosure", The Accounting Review, 72, 275-284, (1997)

Stone,B., "A general class of three-parameter risk measures", Journal of Finance, 29, 675-685, (1973)

Tzur, J., and (Lewinstein) Yaari, V., "Microstructure of firms' disclosure", Review of Quantitative Finance and Accounting, 13, 367-391, (1999)

Woon-Oh Jung and Young K. Kwon, "Disclosure When the market Is Unsure of Information Endowment of managers", Journal of Accounting Research, 26.1, 146-153, (1988). 


\section{Appendix: Proof of the Marginal Values Theorem}

Working from the identity

$$
V(t) \mathbb{P}(N(t))=E_{\mathbb{P}}\left[\mathbb{X} 1_{N(t)}\right]=\int_{N(t)} \mathbb{X} d \mathbb{P}(\omega),
$$

differentiation gives

$$
d V(t) \mathbb{P}(N(t))+V(t) d \mathbb{P}(N(t))=d E_{\mathbb{P}}\left[\mathbb{X} \mathbf{1}_{N(t)}\right]=t d \mathbb{P}(N(t)),
$$

so that

$$
d V(t) \mathbb{P}(N(t))=(t-V(t)) d \mathbb{P}(N(t)) .
$$

Since $d \mathbb{P}(N(t))=q d F(t)$, we can write this explicitly as

$$
V^{\prime}(t)=(t-V(t)) \frac{q}{p+q F(t)} f(t)
$$

where $F(t)$ denotes the cumulative distribution function (for the probability law of the random variable $X$ ) and is assumed to be of the form

$$
F(t)=\int_{0}^{t} f(x) d x
$$

for some continuous $f(x)$. Hence

$$
W^{\prime}(t)=(V(t)-t) \frac{p q}{p+q F(t)} f(t)
$$

